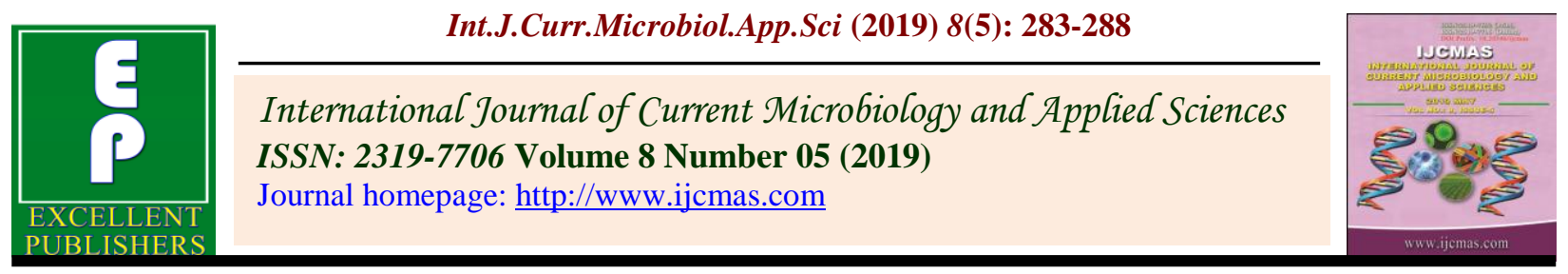

Original Research Article

https://doi.org/10.20546/ijcmas.2019.805.033

\title{
Roving Survey on Banana Diseases in Mysuru District
}

\author{
N. Thammaiah", G.S.K. Swamy, G.K. Sudarshan and Venkat Rao \\ Department of Plant Pathology, College of Horticulture, Mysuru, India \\ *Corresponding author
}

\begin{abstract}
A B S T R A C T
Keywords

Sigatoka leaf spot, Fusarium wilt, Banana, Rhizome rot, Freckle leaf spot

Article Info

Accepted:

04 April 2019

Available Online:

10 May 2019

The roving suvey was conducted during 2016 to December 2017 and January 2018 at different places of Mysuru district on cultivars of Budubale, Ney Poovan, Grand Naine Nanjanagudu Rasabale and Nendran. Sigatoka leaf spot disease appeared in low to moderate form. The disease appeared severe form in Echagahally on Nanjanagudu Rasabale (56.76\%). Freckle leaf spot appeared in low to moderate form. Incidence of Fusarium wilt ranged from 0.0 to $6.0 \%$ in Sept-October 2016, 0.0-2.86\% in February 2017, 0.0-25.86\% in August-September 2017 and 0.0-10.00\% in December 2017- Jan 2018. The bacterial rhizome rot incidence ranged from 0.0 to $21.49 \%$ in $2016,0.0-8.75 \%$ in the month of September-October 2016, 0.0-10.00\% in the month of February 2017, 0.0$21.05 \%$ in the month of August-September 2017 and $0.0-12.79 \%$ in the month of December 2017-January 2018.
\end{abstract}

\section{Introduction}

Banana is one of the important tropical fruit crop. It is widely distributed fruit crop of India. The crop has a wide host range of adoptability as observed by its presence from sea level to a height of $1524.39 \mathrm{~cm}$ and from tropical to subtropical conditions. India is also the home of multitudes of varieties. The varieties were grown with regional preference. Banana is having great socio economic significance in India and it supports livelihood of millions of people with an annual production of $31.08 \mathrm{~m}$ tonnes from 8.59 lakh ha. In Karnataka, banana is grown in 1.01 lakh ha area with total production of $2.4 \mathrm{~m}$. tonnes. In India, banana crop is affected by many diseases. Among these,
Panama disease (Fusarium wilt), Sigatoka leaf spot and rhizome rot are economically important.

\section{Materials and Methods}

Roving survey was conducted during 2016, September-October 2016, February 2017, August-September 2017 and December 2017 -January 2018 at different places of Mysuru district on cultivars of Budubale (ABB), Ney Poovan (AB), Grand Naine (AAA), Nendran (AAA) and Nanjanagudu Rasabale (Rasthali, AAB). Observations were recorded with respect to intensity of Sigatoka leaf spot. A 06 scale was used for scoring the Sigatoka leaf disease index (Gauhl et al., 1993). Where $0=$ No symptoms, $1=$ Less than 1 percent of 
lamina with symptoms (only streaks and / or up to 10 spots), $2=1$ to 5 per cent of lamina with symptoms, $3=6$ to 15 per cent of lamina with symptoms, $4=16$ to 33 per cent of lamina with symptoms, $5=34$ to 50 per cent of lamina with symptoms and $6=51$ to 100 per cent of lamina with symptoms. The freckle leaf spot intensity was recorded. Incidence of Fusarium wilt, rhizome rot and CMV were recorded.

\section{Results and Discussion}

Survey results revealed that Sigatoka leaf spot disease occurrence was low to moderate form and the highest intensity $(27.39 \%)$ was recorded in Harohally. Freckle leaf spot appeared in low to moderate form and freckle leaf spot intensity was highest (19.74\%)in Pura, H.D Kote Tq. Bacterial rhizome rot incidence ranged from 0.0-21.49\%.BBTV incidence was recorded in Yelwala $(2.67 \%)$. BBMV incidence was recorded in Devarasanahally (1.43\%) (Table 1).

In the month of September -October 2016, the Sigatoka leaf spot intensity ranged from $2.00-19.65 \%$ and it was highest $(19.65 \%)$ in Kugulur on Grand Naine. Freckle leaf spot was recorded in Kugulur on Grand Naine $(6.00 \%)$. BBTV was noticed in Kugulur (1.00\%). Fusarium wilt incidence ranged from $0.0-6.00 \%$. The highest incidence of Fusarium wilt was recorded in Kalavadi, Mysore on Budubale $(6.00 \%)$ and Kugulur $(0.44 \%)$.The incidence of bacterial rhizome rot was highest $(8.75 \%)$ in Kadakola followed by Kalawadi $(4.00 \%)$ and Kugulur $(2.44 \%)$. Survey was conducted in the month of November, results revealed that, the intensity of Sigatoka leaf spot was $31.04 \%$ and $4.71 \%$ of rhizome rot (Table 2).

In the month of February 2017, Sigatoka leaf spot intensity ranged from $1.20-15.71 \%$. The intensity of Sigatoka leaf spot was highest $(15.71 \%)$ in Haledidi, Nanjanagudu Tq followed by Kamralli (5.89\%) on Ney Poovan. The highest freckle leaf spot intensity was recorded in Haledidi on Grand Naine (12.50\%) followed by Nagavala on Grand Naine $(3.62 \%)$. Traces of intensity were recorded in Nagavala. Shindehalli and Hallada Manuganahalli on NeyPoovan (Table 3). Incidence of Fusarium wilt ranged from 0.0-2.86\%.CMV was recorded in Tandavapura. The bacterial rhizome rot incidence was highest $(10.00 \%)$ in Alanahalli followed by Shindehalli $(08.00 \%)$ and Tandavapura $(7.66 \%)$.

During the month of August-September 2017, the Sigatoka leaf spot disease appeared low to moderate form. The highest Sigatoka leaf spot index $(40.17 \%)$ was recorded in Devarasanahalli on Nanjanagudu Rasabale followed by Beechanahalli canal $(38.20 \%)$ on Nanjanagudu Rasabale. The incidence of Fusarium wilt ranged from 0.0-25.86\%. Incidence of Fusarium wilt was highest $(25.86 \%)$ in Hullolli on Nanjanagudu Rasabale followed by Devarasanahalli (23.08\%), Beechanahalli $(17.86 \%$ on Nanjanagudu Rasabale and $7.20 \%$ on Ney Poovan). Incidence of rhizome rot ranged from $0.0-21.05 \%$.

The highest incidence of rhizome rot $(21.05 \%)$ was recorded in Hullolli on Nanjanagudu Rasabale followed by Madapur (20.84\%), Beechanahalli canal $(10.0 \%)$ and Devarasanahalli (4.00\%) (Table 4). The cultivars 'Rasthali' (syn. 'Malbhog', 'Nanjangod Rasabale', 'Amrithapani', Martaman', AAB, Silk), 'Karpuravalli' (syn. 'Kanthali', ABB, Pisang Awak), 'Monthan' (ABB) and 'Virupakshi' (syn. 'Hill Banana', $\mathrm{AAB}$, Pome) are severely affected by wilt (Thangavelu et al., 2001).

In Karnataka, cultivation of the local cultivar 'Nanjangod Rasabale' has been reduced from 500 ha to less than 50 ha (Narendrappa and 
Gowda, 1995) due to severe incidence of Fusarium wilt.

In Bihar, more than $55 \%$ of the area under susceptible cultivars was severely infected, and yield reduction in these areas was estimated at $50-70 \%$. In Tamil Nadu, it is becoming a major threat, with disease severity as high as $80-90 \%$ (Sivamani, 1987).

Table.1 Roving survey on banana diseases in Mysuru (2016)

\begin{tabular}{|c|c|c|c|c|c|c|c|}
\hline Place & Cultivar & $\begin{array}{c}\text { Sigatoka } \\
\text { leaf spot } \\
\text { index }\end{array}$ & $\begin{array}{c}\text { Freckle } \\
\text { leaf spot } \\
\text { index }\end{array}$ & $\begin{array}{l}\text { Fusarium } \\
\text { Wilt (\%) }\end{array}$ & $\begin{array}{c}\text { BBTV } \\
(\%)\end{array}$ & $\begin{array}{c}\text { BBMV } \\
(\%)\end{array}$ & $\begin{array}{c}\text { Rhizome } \\
\text { rot }(\%)\end{array}$ \\
\hline $\begin{array}{l}\text { Yelwala, } \\
\text { Hunsur Tq }\end{array}$ & Grand Naine & 6.55 & 16.62 & 0.0 & 2.67 & 0.0 & 1.60 \\
\hline \multirow{2}{*}{$\begin{array}{l}\text { Harohalli, } \\
\text { Mysuru }\end{array}$} & Ney Poovan & 2.12 & 9.04 & 0.0 & 0.0 & 0.0 & 21.49 \\
\hline & Grand Naine & 27.39 & 1.72 & 0.0 & 0.0 & 0.0 & 0.0 \\
\hline $\begin{array}{l}\text { Kadakola, } \\
\text { Nanjanagudu Tq }\end{array}$ & Ney Poovan & 1.98 & 0.0 & 0.0 & 0.0 & 0.0 & 5.05 \\
\hline $\begin{array}{l}\text { Devarasahally, } \\
\text { Nanjanagudu Tq }\end{array}$ & Ney Poovan & 2.83 & Traces & 0.0 & 0.0 & 1.43 & 0.0 \\
\hline \multirow{2}{*}{$\begin{array}{l}\text { Pura, } \\
\text { H.D.Kote Tq }\end{array}$} & Ney Poovan & 5.99 & 0.0 & 0.0 & 0.0 & 0.0 & 2.73 \\
\hline & Nendran & 5.60 & 19.74 & 0.0 & 0.0 & 0.0 & 0.0 \\
\hline
\end{tabular}

Table.2 Roving survey on banana diseases in Mysuru district (September-October 2016)

\begin{tabular}{|c|c|c|c|c|c|c|}
\hline Place & Cultivar & $\begin{array}{c}\text { Sigatoka leaf } \\
\text { spot } \\
\text { Index } \\
\text { (M.musicola) }\end{array}$ & $\begin{array}{c}\text { Freckle leaf spot } \\
\text { index } \\
\text { (Phyllostictina } \\
\text { musarum) }\end{array}$ & $\begin{array}{c}\text { BBTV } \\
(\%)\end{array}$ & $\begin{array}{c}\text { Fusarium } \\
\text { wilt }(\%)\end{array}$ & $\begin{array}{c}\text { Bacterial } \\
\text { Rhizome rot } \\
(\%) \\
\text { (Erwinia spp.) }\end{array}$ \\
\hline \multirow[t]{3}{*}{$\begin{array}{c}\text { Kalavadi, Mysuru } \\
\mathbf{T q}\end{array}$} & $\begin{array}{l}\text { Budubale } \\
\text { (ABB) }\end{array}$ & 2.00 & 0.00 & 0.00 & 6.00 & 0.00 \\
\hline & $\begin{array}{c}\text { Ney Poovan } \\
\text { (AB) }\end{array}$ & 3.00 & 0.00 & 0.00 & 0.00 & 4.00 \\
\hline & Grand Naine & 10.13 & 0.00 & 0.00 & 0.00 & 0.00 \\
\hline \multirow[t]{2}{*}{$\begin{array}{c}\text { Kuguluru, } \\
\text { Nanjanagudu Tq }\end{array}$} & $\begin{array}{l}\text { N.Rasabale } \\
\text { (AAB) }\end{array}$ & 10.73 & 0.00 & 1.00 & 0.44 & 2.44 \\
\hline & $\begin{array}{c}\text { Grand Naine } \\
\text { (AAA) }\end{array}$ & 19.65 & 6.00 & 0.00 & 0.00 & 0.00 \\
\hline $\begin{array}{c}\text { Kadakola, } \\
\text { Nanjanagudu Tq }\end{array}$ & $\begin{array}{l}\text { Ney Poovan } \\
\text { (AB) }\end{array}$ & 8.05 & 0.00 & 0.00 & 0.00 & 8.75 \\
\hline $\begin{array}{c}\text { Nov.2017 } \\
\text { Manuganahall, } \\
\text { H.D.Kote Tq }\end{array}$ & $\begin{array}{l}\text { Grand Naine } \\
\text { (AAA) }\end{array}$ & 31.04 & 0.00 & 0.00 & 0.00 & 4.71 \\
\hline
\end{tabular}


Table.3 Roving survey on banana diseases in Mysuru district (February 2017)

\begin{tabular}{|c|c|c|c|c|c|c|}
\hline Place & Cultivar & $\begin{array}{c}\text { Sigatoka leaf } \\
\text { spot } \\
\text { Index } \\
\text { (M.musicola) }\end{array}$ & $\begin{array}{c}\text { Freckle leaf } \\
\text { spot index } \\
\text { (Phyllostictina } \\
\text { musarum) }\end{array}$ & $\begin{array}{c}\text { Fusarium } \\
\text { wilt }(\%)\end{array}$ & $\begin{array}{c}\text { CMV } \\
(\%)\end{array}$ & $\begin{array}{c}\text { Bacterial } \\
\text { Rhizome rot } \\
(\%) \\
\text { (Erwinia spp.) }\end{array}$ \\
\hline \multirow[t]{2}{*}{$\begin{array}{l}\text { Nagavala, } \\
\text { Mysuru Tq }\end{array}$} & $\begin{array}{c}\text { Grand } \\
\text { Naine }(\mathrm{AAB})\end{array}$ & 5.68 & 3.62 & 0.00 & 0.00 & 0.00 \\
\hline & $\begin{array}{l}\text { Ney Poovan } \\
\text { (AB) }\end{array}$ & 2.69 & Traces & 0.13 & 0.00 & 0.25 \\
\hline $\begin{array}{l}\text { Kamaralli, } \\
\text { Mysuru Tq }\end{array}$ & $\begin{array}{l}\text { Ney Poovan } \\
\text { (AB) }\end{array}$ & 5.89 & 0.00 & 0.00 & 0.00 & 4.00 \\
\hline $\begin{array}{l}\text { Shindehalli, } \\
\text { H.D.Kote Tq. }\end{array}$ & $\begin{array}{l}\text { Ney Poovan } \\
\text { (AB) }\end{array}$ & 4.72 & Traces & 2.86 & 0.00 & 8.00 \\
\hline $\begin{array}{l}\text { Alanahalli, } \\
\text { H.D.Kote Tq. }\end{array}$ & $\begin{array}{c}\text { NeyPoovan } \\
\text { (AB) }\end{array}$ & 3.84 & 0.00 & 0.00 & 0.00 & 10.00 \\
\hline $\begin{array}{c}\text { Hallada } \\
\text { Manuganahalli } \\
\text {,H.D.Kote Tq }\end{array}$ & $\begin{array}{l}\text { Ney Poovan } \\
\text { (AB) }\end{array}$ & 1.47 & Traces & 1.00 & 0.00 & 1.00 \\
\hline $\begin{array}{c}\text { Tandavapura, } \\
\text { Nanjanagudu } \\
\text { Tq }\end{array}$ & $\begin{array}{l}\text { Ney Poovan } \\
\text { (AB) }\end{array}$ & 1.20 & 0.00 & 0.00 & 0.06 & 7.66 \\
\hline $\begin{array}{c}\text { Haledidi, } \\
\text { Nanjanagudu } \\
\text { Tq }\end{array}$ & $\begin{array}{c}\text { Grand } \\
\text { Naine(AAB }\end{array}$ & 15.71 & 12.50 & 0.00 & 0.00 & 0.00 \\
\hline
\end{tabular}

Table.4 Survey on banana diseases in Mysuru district-August-September 2017

\begin{tabular}{|c|c|c|c|c|c|c|}
\hline Place & Cultivar & $\begin{array}{c}\text { Sigatoka leaf spot } \\
\text { index } \\
\text { (Mycosphaerella } \\
\text { musicola) }\end{array}$ & $\begin{array}{c}\text { Freckle leaf } \\
\text { spot index } \\
\text { (Phyllostictina } \\
\text { musarum) }\end{array}$ & $\begin{array}{c}\text { Fusarium } \\
\text { wilt } \\
(\%)\end{array}$ & $\begin{array}{c}\text { BBTV } \\
(\%)\end{array}$ & $\begin{array}{c}\text { Rhizome } \\
\text { rot }(\%) \\
\text { Erwinia } \\
\text { spp }\end{array}$ \\
\hline \multirow[t]{3}{*}{ Hullolli } & $\begin{array}{c}\text { Rasthali } \\
\text { (N.Rasabale AAB) }\end{array}$ & 7.68 & 0.00 & 25.86 & 0.00 & 21.05 \\
\hline & Ney Poovan & 3.64 & 0.00 & 0.00 & 0.00 & 5.00 \\
\hline & $\begin{array}{c}\text { Rasthali } \\
\text { (N.Rasabale } \\
\text { AAB) }\end{array}$ & 1.81 & 0.00 & 5.56 & 0.00 & 0.00 \\
\hline Madapura & $\begin{array}{c}\text { Rasthali } \\
\text { (RasabaleAAB) }\end{array}$ & 6.37 & 0.00 & 1.00 & 0.00 & 20.84 \\
\hline Hadaginalu & Ney Poovan(AB) & 7.83 & 0.00 & 0.00 & 0.00 & 0.00 \\
\hline \multirow[t]{3}{*}{ Devarasanahalli } & Ney Poovan(AB) & 10.18 & 0.00 & 1.52 & 0.00 & 4.00 \\
\hline & $\begin{array}{c}\text { Rasthali } \\
\text { (N.Rasabale AAB) }\end{array}$ & 40.17 & 0.00 & 23.08 & 0.00 & 0.00 \\
\hline & Veg.type & 29.17 & 0.00 & 0.00 & 0.00 & 0.00 \\
\hline \multirow{2}{*}{$\begin{array}{l}\text { Beechanahalli } \\
\text { (canal) }\end{array}$} & Ney Poovan(AB) & 6.75 & 0.00 & 7.20 & 0.00 & 5.26 \\
\hline & $\begin{array}{c}\text { Rasthali } \\
\text { (N.Rasabale AAB) }\end{array}$ & 38.20 & 0.00 & 17.86 & 0.00 & 10.00 \\
\hline
\end{tabular}


Table.5 Survey on banana diseases in Mysuru district-December2017-January 2018

\begin{tabular}{|c|c|c|c|c|c|c|}
\hline Place & Cultivar & $\begin{array}{l}\text { Sigatoka leaf } \\
\text { spot index } \\
\text { (Mycosphaere } \\
\text { lla musicola) }\end{array}$ & $\begin{array}{c}\text { Freckle leaf } \\
\text { spot index } \\
\text { (Phyllostictina } \\
\text { musarum) }\end{array}$ & $\begin{array}{c}\text { Fusarium } \\
\text { wilt (\%) }\end{array}$ & $\begin{array}{c}\text { BBTV } \\
(\%)\end{array}$ & $\begin{array}{c}\text { Rhizome } \\
\text { rot }(\%) \\
\text { Erwinia } \\
\text { spp }\end{array}$ \\
\hline \multirow[t]{3}{*}{$\begin{array}{l}\text { Echagahalli, } \\
\text { Nanjanagudu Tq }\end{array}$} & $\begin{array}{l}\text { Rasthali } \\
\text { (N.Rasabale } \\
\text { AAB) }\end{array}$ & 56.76 & 0.00 & 1.00 & 0.00 & 0.00 \\
\hline & $\begin{array}{l}\text { Ney } \\
\text { Poovan(AB) }\end{array}$ & 6.61 & 0.00 & 0.00 & 0.00 & 1.00 \\
\hline & $\begin{array}{l}\text { Grand } \\
\text { Naine(AAA) }\end{array}$ & 45.04 & 0.00 & 0.00 & 0.00 & 0.00 \\
\hline $\begin{array}{l}\text { Sinduvallipura } \\
\text { Nanjanagudu Tq }\end{array}$ & $\begin{array}{l}\text { Ney } \\
\text { Poovan(AB) }\end{array}$ & 8.42 & 0.00 & 0.00 & 0.00 & 6.21 \\
\hline $\begin{array}{l}\text { Yelachagere } \\
\text { Nanjanagudu Tq }\end{array}$ & $\begin{array}{l}\text { Ney } \\
\text { Poovan(AB) }\end{array}$ & 12.56 & 0.00 & 7.50 & 0.00 & 0.00 \\
\hline $\begin{array}{l}\text { Tandavapura } \\
\text { Nanjanagudu Tq }\end{array}$ & $\begin{array}{l}\text { Ney } \\
\text { Poovan(AB) }\end{array}$ & 6.03 & traces & 0.00 & 0.00 & 12.79 \\
\hline \multirow[t]{2}{*}{$\begin{array}{l}\text { Kogaluru } \\
\text { Nanjanagudu Tq }\end{array}$} & $\begin{array}{l}\text { Ney } \\
\text { Poovan(AB) }\end{array}$ & 2.20 & 0.00 & 0.00 & 2.25 & 1.25 \\
\hline & Grand Naine & & & & & 5.00 \\
\hline Mobballi & $\begin{array}{l}\text { Ney } \\
\text { Poovan(AB) }\end{array}$ & 5.01 & 0.00 & 3.13 & 0.00 & 4.00 \\
\hline \multirow[t]{2}{*}{ Heggadahalli } & $\begin{array}{l}\text { Ney } \\
\text { Poovan(AB) }\end{array}$ & 9.05 & 0.00 & 10.00 & 0.00 & 2.00 \\
\hline & $\begin{array}{l}\text { Grand } \\
\text { Naine(AAA) }\end{array}$ & 30.00 & 0.00 & 0.00 & 0.00 & 0.00 \\
\hline $\begin{array}{l}\text { Kembala } \\
\text { (Mysuru) }\end{array}$ & $\begin{array}{l}\text { Ney } \\
\text { Poovan(AB) }\end{array}$ & 1.61 & 0.00 & 6.00 & 0.00 & 1.00 \\
\hline $\begin{array}{l}\text { Rayanakere } \\
\text { (Mysuru) }\end{array}$ & $\begin{array}{l}\text { Ney } \\
\text { Poovan(AB) }\end{array}$ & 4.62 & 0.00 & 7.00 & 0.00 & 0.00 \\
\hline
\end{tabular}

In the months of December 2017-January 2018 ,the Sigatoka leaf spot disease appeared in low to moderate form. The Sigatoka leaf spot index was highest in Echagahalli $56.76 \%$ on Nanjanagudu Rasabale and $45.04 \%$ on Grand Naine) followed by Heggadahalli on Grand Naine $(30.0 \%)$ and Yelachagere (12.56\%). The freckle leaf spot disease appeared traces in Tandavapura Incidence of Fusarium wilt ranged from $0.0-10.0 \%$. The highest incidence of Fusarium wilt $(10.0 \%)$ was recorded in Heggadahalli followed by Yelachagere (7.50\%), Rayanakere (7.00\%), Kembala (6.00\%),
Mobballi (3.13\%) and Echagahalli (1.00\%).The incidence of BBTV recorded in Kugulur $(2.25 \%)$. Incidence of rhizome rot ranged from $0.0-12.79 \%$. The highest incidence was recorded in Tandavapura (12.79\%) followed by Sinduvallipura $(6.21 \%)$, Kuguluru $(5.00 \%)$ and Mobballi (4.00\%) (Table 5). Sigatoka leaf spot was reported for the first time in India (Jones, 2000). Survey was conducted during revealed that almost all the commercial cultivars in Kerala were affected by Sigatoka leaf spot (Estelitta et al., 1996). The prevalence of freckle leaf spot was reported at Coimbatore, 
Trichy, Periyar and North Arcot districts of Tamilnadu during 1995-96 (Anon.1997). A roving survey was conducted to assess the severity of tip-over disease of Banana in the farmer's field of north eastern Karnataka. The highest disease incidence $(20.35 \%)$ was recorded at Muddaballi village of Koppal district. The least incidence (1.15\%) was recorded in Ankammanahal village of Sandur taluk of Bellary district. Taluk wise mean incidence revealed that, highest disease incidence of 17.92 per cent was recorded in Koppal followed by Shimoga (12.0\%), Gangavati $(8.07 \%)$, Holalkere $(7.8 \%)$ and Sagar $(6.30 \%)$ taluks. The minimum incidence of 1.75 per cent was recorded in Sandur, Hospet (4.69\%), Hiriyur $(5.7 \%)$ taluks. District-wise, highest tip over incidence of 12.99 was recorded from Koppal followed by Shimoga $(9.15 \%)$ and Chitrdurga (6.75\%). Lowest incidence of 3.22 per cent was recorded in Ballari district of Karnataka. Nagaraj et al., (2012) reported the incidence of tip over ranged from 30-35 per cent in the districts of Bangalore and Kolar of Karnataka state.

\section{References}

Anonymous, 1997, Research Report (Citrus, Banana, Papaya, Pineapple, and Sapota). Group discussion, UAS, Dharwad, 27-30 Nov. 1997. Pp. 386, 388, 391-392.

Anonymous, 2017-2018, NHB.

Estelitta, S., Suma, A. and Pushkaran, K., 1989, Assessment of losses caused by leaf spot of banana. Annual Research Report, 1988-89. Banana Research station, Kannara, Kerala Agricultural University.

Gauhl, F., Pasberg-Gauhl, C., Vuylsteke, D. and Ortiz, R, 1993, Multilocation evaluation of black Sigatoka resistance in banana and plantain. International Institute of Tropical Agriculture (IITA), Research Guide, 47,
Ibudan, Nigeria, pp.59.

Jones, 2000, Sigatoka. In diseases of banana, abaca and enset. Ed. Jones, D.R. Commen wealth Agril. bureau internati zonal.pp.7992.

Nagaraj, M.S., Umashankar, N., Palanna, K. B and Khan, A.N.A. etiology and management of tip-over disease of banana by using biological agents. Int.J.Advanced .Biological Research., 2(3)2012:483-486.

Narendrappa, T., and Gowda, B.J. 1995. Integrated management of Panama wilt on banana cv. Nanjangud Rasabale. Current Research, University of Agricultural Sciences, Bangalore 24:53-55.

Saranavan, T., Muthusamy, M., and Marimuthu, T., 2003. Development of integrated approach to manage the fusarial wilt of banana. Crop Protection. 22:1117-1123.

Sivamani, E., 1987. Wilt diseases of banana in Tamil Nadu. Studies on the biology and control of Panama wilt caused by Fusarium oxysporum f. sp. cubense(E.F. Smith) Snyder ed. Hansen. Ph.D. Thesis. University of Madras, Chennai, India.

Thangavelu, R., Sundararaju, P., Sathiamoorthy, S., Raghuchander, T., Velazhahan, R., Nakkeeran, S. and Palaniswami, A. 2001. Status of Fusarium wilt of banana in India. p.58-63.

Thiyagarajan, V., S.T. Yenjerappa, Gururaj Sunkad, D.S. Aswathanarayana, V. Karuppaiah and H.M. Shaila. 2017. Survey on Tip-over Disease of Banana caused by Erwinia carotovora subsp. carotovora (Jones) Holland in Parts North Eastern Karnataka, India. Int.J.Curr.Microbiol. App.Sci (2017) 6(6): 2973-2976.

Saranavan, T., Muthusamy, M., and Marimuthu, T., 2003. Development of integrated approach to manage the fusarial wilt of banana. Crop Protection. 22:1117-1123.

\section{How to cite this article:}

Thammaiah, N., G.S.K. Swamy, G.K. Sudarshan and Venkatrao. 2019. Roving Survey on Banana Diseases in Mysuru District. Int.J.Curr.Microbiol.App.Sci. 8(05): 283-288.

doi: https://doi.org/10.20546/ijcmas.2019.805.028 